\title{
OBSERVATIONS ON VACCINIAL ERUPTIONS AND DIFFERENTIAL DIAGNOSIS OF GENERALIZED VACCINIA
}

\author{
JuAn J. Angulo, M.D. (Havana) \\ Epidemiologist \\ Luis F. de Salles-Gomes, M.D. (São Paulo)
Epidemiologist
}

Instituto Adolfo Lutz, São Paulo, Brazil

Thales de Britto, M.D. (São Paulo)

Pathologist

Faculdade de Medicina, Universidade de São Paulo, São Paulo

\author{
Cacilda Cubas dos Santos, M.D. (São Paulo) \\ Orlando N. Bassoi, M.D. (São Paulo) \\ Physician \\ Hospital das Clinicas, São Paulo \\ Physician
}

IN spite of literature on generalized vaccinia, the differential diagnosis of this condition has received little attention while the clinical picture of eruptions following vaccination against smallpox continuously offers field for new findings. In the present paper the relevant characteristics of four cases of laboratory-confirmed vaccinial eruption are recorded and some reflections on the differential diagnosis of generalized vaccinia are made after observation of more than 50 cases.

\section{Case I-Eczema Vaccinatum}

\section{(Kaposi's Varicelliform Eruption)}

A 21-year-old Negro woman had been admitted to the Pemphigus Foliaceus Hospital of São Paulo City one year before the present observation because of a generalized atopic dermatitis. The latter had its onset one year after birth and persisted until her examination made 20 years later. The patient had been vaccinated with ' take' when she was eight years old but there was no generalization of vaccinia. On December 13, 1955, she was vaccinated along with numerous other hospital inmates. A high percentage of vaccinated patients as well as a number of those not vaccinated presented generalized vaccinia. The patient did not develop a 'take', nor show generalized vaccinia. Twenty-four days later, the majority of the remaining inmates were vaccinated and this provoked another outbreak of generalized vaccinia. On January 25, 1956, when most of these cases were recovering from vaccinial lesions, the patient showed systemic manifestations including headache, general malaise, prostration and fever. After three days of these manifestations, a scanty eruption composed of vesicular lesions appeared. These pocks, numbering about two dozen, were localized on the extremities and buttocks; numerous lesions soon appeared all over the body. The pocks became pustules with the appearance typical of variola vera lesions since they were convex, hemispheric whiteish or creamish pocks undergoing umbilication. The chest exhibited, in addition to numerous pocks, small areas of skin denudation with congested bases. Facial œdema was prominent. The systemic manifestations of the prodromic period continued for the first week of the exanthem. The clinical course was uneventful and no modification of the atopic dermatitis was noticed.

Laboratory findings. The techniques employed in the identification of this and the other cases reporte 4 here mainly were those developed by Downie an associates (Downie and Dumbell, 1947; McCarthy and Downie, I 953; Kempe, 1956). The "Instituto Butantafe strain of vaccinia virus was used in the preparation of antigens for serological tests and in the neutralization test. Two specimens, each consisting of the contents of several pustules, were inoculated on the chorioallantois of chick embryos (Downie and Dumbell, r947). Vaccinia virus was isolated and identified (Downie and Dumbell, 1947; Kempe, 1956) from both specimens. Complement fixation (McCarthy and Downie, 1953) titers of $I: 1280$ were found in each of two serum specimens collected five days and eight days, respectively, after the onset of the illness. In the same specimens, the hæmagglutination inhibition test (McCarthy and Downie, 1953) yielded titers of $1: 320$ and $I: 5120$, respectively.

Comment. The superimposition of a pock-rash on the atopic dermatitis composed a clinical picture usually known as Kaposi's varicelliform eruption, but it might as well be called eczema vaccinatum. The pock-rash presented such a close similarity with variolous eruptions that a clinician with ample experience in variola and generalized vaccinia identified it, without hesitation, as a smallpox exanthem. Incidentally, the outbreaks of generalized vaccinia had disappeared when this case was examined. The pock-distribution was so widespread that the asymmetry and accumulation of lesions characterizing many of the other cases occurring during the outbreaks were not present. In addition, the morphology of individual pocks closely mimicked that of classical variolous lesions. The patient showed a definite prodromal period whose symptomatology and duration made the diagnosis of smallpox more likely since cases of generalized vaccinia not infrequently show few if any manifestations before the onset of the exanthem. In an overall appraisal, the distribution of pocks was rather uniform because neither centrifugal 
nor centripetal localization of pocks was clearly apparent, a finding which may be made in smallpox cases with profuse efflorescences. As usually found in variola, there were fewer pocks on the forearm than on the back of the hand, but more lesions were seen on the arm than on the forearm and this did not suggest smallpox. The absence of pocks from the palms and soles was definitely against smallpox.

The vaccinial ætiology of the exanthem was satisfactorily established by recovery of the virus from two specimens obtained from numerous skin lesions. This result left no doubt about the absence of variola virus from these specimens, since serial passaging and reinoculations of the latter did not show the occurrence of variola-like lesions. The high titers obtained in the serological tests constitute supporting evidence, but have no value for the differentiation between variolous and vaccinial infections because of known antigenic relationships between these viruses (McCarthy and Downie, I953). In summary, the case acquired the vaccinia infection through contagion, since her inoculation in the first mass vaccination failed and she apparently was a case secondary to some of the cases provoked by the second mass vaccination. Not only the absence of a ' take' discourages the hypothesis of the generalized vaccinia being due to her inoculation in the first mass vaccination but the incubation period would be, in this case, 43 days, obviously too long. The occurrence of the vaccinial eruption was apparently influenced by the previously existing condition, namely atopic dermatitis. Incidentally, the generalized distribution of the latter apparently determined the equally generalized vaccinial exanthem, thus contributing to the close similarity with smallpox.

\section{Case 2-Progressive Vaccinia (Prolonged}

\section{Generalized Vaccinia, Gangrenous Vaccinia)}

A.R., a four-month-old white boy, was vaccinated on April 13, 1954. Ten days later, the 'take' was showing an abnormal appearance and a scarlatiniform rash appeared on the trunk and then on the face, but it had a short duration. Twenty-three days after vaccination the 'take' was not in regression but was larger than on the tenth day. A few pocks then appeared around the left ear as well as on the place, in the neck, where an intravenous injection had been made. Two days later, some pocks were noticed on the buttocks and genitalia. By the time of death, 39 days after vaccination, the 'take' had coalesced with satellite pocks forming a phægedenic ulcer, as also were other lesions on the scrotum, ears, neck and buttocks. A few scattered pustules were found around these ulcers. The lesions on the lips and nose extended into the buccal and nasal mucosæ. Hæmorrhagic crusts were found on ulcerated lesions.

Laboratory findings. The histopathological study of autopsy material depicted degeneration and necrosis of the epidermis, while the dermis exhibited infiltration by histiocytes, lymphocytes and some eosinophils. Periportal areas of hyaline necrosis were found in the liver as well as diffuse congestion and fatty degeneration, focal hæmorrhages and lymphocyte infiltrations, showing also some histiocytes and eosinophils. The lungs exhibited congestion, cedema and, particularly, foci of necrosis surrounded by hæmorrhages but without definite inflammatory reaction. No other important pathological finding was made apart from small focal hæmorrhages and cdema in the brain and hyperplasia of the splenic pulp. Guarnieri inclusion bodies were found in the epidermis but not in other organs.

Vaccinia virus was isolated from secondary pocks and its identity established through serial passaging and re-inoculations of the chick-embryo chorioallantoisat inoculation of rabbit skin and cornea, a cross-immunity test in rabbits and a neutralization test in chick embryos In the latter two tests, the 'Instituto Butantan' strain of vaccinia virus was employed as the standard virus. $c$

Comment. 'Progressive', or 'prolonged', or 'gan grenous' vaccinia is a clinical entity whose typica $\overline{\sqrt{5}}$ picture was found in the present case. Rather than a tendency to recover and heal, lesions followed a torpid progressive course and were frankly gangrenous. These. characteristics were also found in the 'take'. The fatal end was also typical of this clinical entity. Recovery of vaccinia virus from two specimens of pustula? material and the presence of Guarnieri inclusion bodies in epidermis sections left no doubt about the nature of the pock-lesions following vaccination. The brief erythematous rash appearing on the roth day after vaccination probably was roseola vaccinica.

The peculiar course of progressive vaccinia has been attributed to agammaglobulinemia, but this has not been confirmed, while failure to produce antibodies is generally considered as a determining factor (Kempew. Berge and England, 1956). Regarding the hypothesis of generalization of vaccinia being due to scratching with contaminated fingers (Sommerville, Napier and Dick, I95I), it may be remarked that lesions were found, in the present case, at places on the back wheres scratching by a four-month-old cachectic child iso unlikely. Worthy of mention is the absence of a history of allergy in the patient and his family, since generaliza- tion of vaccinia has been found associated with allergy (Davidson and Davies, r943). Two brothers and his parents had recently been vaccinated and a norma $B$ 'take' had occurred in all instances. Furthermose, $\overrightarrow{0}$ the child had not shown any skin lesions before vis cination. The pathological findings were very simiar to those made by Hall, Cunliffe and Dudgeon (1953) Incidentally, clinical signs of lung involvement occurredo before vaccination.

\section{Case 3-'Inoculation' Vaccinia and Vacciniole}

A ro-year-old white boy was vaccinated against $\frac{\mathbb{Q}}{2}$ smallpox. Two days later, he had malaise and a slight rise in temperature as well as swelling of lymph glands? in the corresponding axilla. A pronounced temperaturerise accompanied by intense agitation later occurred? which subsided on the next day when a pock appearedo on the buccal mucosa. The vaccination 'take' was rather large and surrounded by a strikingly extensive area of inflammation when the patient was examined. Two smaller, 'satellite' pocks were noticed close to $\frac{-}{3}$ the 'take' and the oral pock closely resembled a vac-그. cination pustule. Vaccinia virus was isolated and identified from the buccal lesion as well as from the saliva.

Comment. The patient was inoculated with vaccinia음 virus for vaccination purposes and developed a 'take'.$D$ The latter became complicated by lesions at places은 other than the vaccination site: one in the mouth, 'inoculation' or 'autoinoculation' vaccinia, and two in the immediate vicinity of the "take', vacciniola or" 'satellite' lesions ('Nebenpocken'). The presence of 0 the virus in the saliva was due either to the fact that ${ }_{\mathrm{W}}$ the oral pock had lost its roof, thus exposing its contents, $\sigma$ or to excretion through the saliva of blood-borne virus (Gins, Hackenthal and Kamentzewa, 1929).

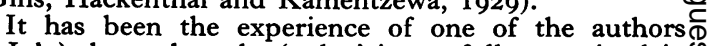
(J.J.A.) that, when the 'take' is carefully examined in? cases with a complication of vaccination such as an $\square$ encephalitis or a vaccinial eruption, the 'take' itself $\overline{0}$ shows anomalies such as an abnormal inflammation of $\frac{\vec{D}}{1}$ the surrounding area, purpuric lesions or a gangrenous 
tendency and its course is more prolonged than usual. Furthermore, temporary remissions of the 'take' were followed by simultaneous remissions of the complication.

Case No. 4-Eczema Vaccinatum and Impetigo

A nine-month-old white boy had been suffering from typical infantile eczema for six months when he presented with a pock eruption. The pocks were vesicles rapidly becoming umbilicated pustules. The eczematous patches were located on the face, shoulders, buttocks, elbows, hands, wrists and ankles. It was precisely in these regions where the pock-eruption appeared. These pocks contained vaccinia virus as shown by laboratory procedures. Impetiginization of the lesions of the face and swelling of cervical lymph nodes followed. Other crops of pocks appeared but the vaccinial complication ended in about ro days. Eczematous lesions continued to appear and they were sampled for virus recovery with negative results. The complement fixation test was positive in the three blood specimens collected during the clinical course. The child had never been vaccinated.

Comment. The patient was suffering from infantile eczema and asthma and had an allergic family background. After contagion from an unknown source, the child developed a vaccinial eruption which complicated the chronic skin condition. Impetiginization of face lesions was a further complication. The striking association of the vaccinial rash with the eczematous areas was obvious because of the sharp limits of the latter. Large cutaneous areas free of lesions of any kind were seen. The identity of the pock eruption was thoroughly established through virus isolation supported by serologic data.

\section{The Differential Diagnosis of Generalized Vaccinia}

Clinical diagnosis of generalized vaccinia may be a difficult task. This is especially true when the eruption appears in a patient with a previous chronic condition of the skin since the latter ailment contributes much to confusion, as in Case No. I. Pock exanthems appearing on previously damaged skin give rise to a clinical picture known as Kaposi's varicelliform eruption whose identity has been the subject of much controversy (Hershey and Smith, I945; Fries, Borne and Barnes, 1948; Sexton, Labzoffsky and Ross, 1957). The latter is partially due to the fact that vaccinia virus (Fries and others, 1948; Sommerville and others, 195I), herpes simplex virus (Barton and Brungsting, 1944; Wenner, 1944) and perhaps other agents (Hershey and Smith, 1945; Sexton and others, I957) may be responsible for the acute pock-rash, which is a component of that syndrome. Furthermore, clinical differentiation between cases of Kaposi's varicelliform eruption whose exanthem is caused by vaccinia virus and those provoked by herpes simplex virus is often impossible because of the morphologic similarity of pocks. Besides, the pock-eruption usually is not varicelliform, as the name implies, but vacciniform or varioliform.

An appraisal of the clinical observations made in more than 50 cases of generalized vaccinia, the majority occurring in patients with pemphigus foliaceus, as well as a review of the literature, led to the conclusion that in a number of cases of generalized vaccinia, the differentiation from smallpox and chickenpox, the most closely related exanthematous diseases, can be made with some accuracy on the basis of clinical characteristics. Besides being limited to these diseases, the present discussion will only deal with significant characteristics and favourable cases.

Many cases of generalized vaccinia do not exhibit a prodromal period, or the latter is illdefined and/or short-lasting. The same is observed, however, in chickenpox but not in smallpox, whose prodromal period has a uniform composition and duration and constantly occurs even in benign cases. In the present series a prodromal period could only be demonstrated in three out of the nine cases whose dates of onset of rash and systemic manifestations were known. Case No. I (see above) is among these three cases. It seems that a prodromal period of generalized vaccinia is more likely to occur in patients with a previous skin condition than in those with an apparently normal skin (Curth, Curth and Garb, 1948).

The pock distribution sometimes offers characteristics having differential value. While many cases of smallpox show a centrifugal distribution of pocks and a number of chickenpox cases a centripetal one, cases of generalized vaccinia do not exhibit a constant or peculiar pattern. Further more, when the patient suffers from a chronic, pre-existing skin condition, the vaccinial eruption exhibits a definite tendency to follow the pattern of that condition. For instance, if the distribution of atopic dermatitis lesions is centrifugal, a vaccinial exanthem in such cases closely simulates smallpox, as happened in a case studied by two of the authors (Angulo and de Salles-Gomes). Besides, if the pre-existing skin condition has a uniform distribution, the superimposition of an extensive vaccinial eruption may show similarities with profuse variolous exanthems so as to make confusion easy, even by experienced clinicians. This happened with Case No. I. Smallpox and chickenpox lesions also are more numerous in skin areas with a local disturbance (Schamberg and Kolmer, 1928), although their distribution in other regions is apparently not altered. Thus, when considering the distribution of skin lesions in differential diagnosis, accumulation in such areas should be neglected when distribution on normal skin is definitely centrifugal or centripetal and the weight of evidence is on the side of smallpox or chickenpox. However, the possibility of a mixed infection actually exists (Angulo and de Salles-Gomes) and should not be overlooked. On the other hand, a diagnosis of smallpox or chickenpox is discouraged if pocks are only found 
in skin areas with a local disturbance and are missing in those usually involved in variolous and varicellous exanthems.

The distribution of vaccinial pocks frequently shows a characteristic which is peculiar to vaccinia and thus conclusive for differentiating it from smallpox although not from chickenpox. This characteristic is the absence of pocks from the palms and/or soles in cases with profuse exanthem. Case No. I, who exhibited an extremely abundant vaccinial eruption uniformly distributed on the body surface, did not show palmar or plantar lesions. Only one case showed these lesions on the palms although none was found on the soles among 29 cases from the present series. Palmar and plantar pocks are absent in some very scanty variolous eruptions, but this finding is common in chickenpox.

Restriction of the eruption to certain body areas may have a differential significance in some cases since the pocks of variolous exanthems are usually widespread even in scanty rashes. This difference is more marked and definitely significant when a large number of pocks accumulate in a few body regions leaving the neighbouring areas free, as may happen in cases of generalized vaccinia previously suffering from certain skin conditions. In this regard a case of eczema vaccinatum reported by one of the authors (de Salles-Gomes, Bassoi and Patricio, I955) showed such numerous vaccinial pocks in the popliteal regions that they touched each other while the rest of the body was completely free even in the neighbourhood of the eczematous patches. Another case of eczema vaccinatum observed by two of the authors (Angulo and de Salles-Gomes) exhibited a very profuse eruption on the head and extremities, while the trunk was strikingly free. This phenomenon has been noticed by other workers (Fries and others, 1948; Sommerville and others, I95I) and it is not expected to occur in profuse smallpox or chickenpox eruptions, while it can hardly be observed in scanty variolous exanthems.

While many varicellous and variolous eruptions essentially are symmetrical because of an equal or almost equal distribution on the right and left body halves, vaccinial eruptions sometimes are clearly more profuse and/or involve larger areas on one side of the body than on the other. Several cases from the present series showed this distribution. It was particularly striking in some vaccinated patients whose body-half corresponding to the vaccinated arm showed more lesions than the opposite half. In some of these cases asymmetrical distribution was so marked that lesions had appeared only on one body-half. For instance, one case showed secondary pocks exclusively on the vaccinated arm, while another case exhibited vaccinial lesions on the vaccinate arm and on one side of the face. The asymmetr in some cases was also apparent in regard to con $\bar{Q}$ fluence of pocks which sometimes occurred on one side of the body, while on the other pocks: were separate. When present, the asymmetr and especially the unilaterality of skin lesions are almost conclusive and conclusive, respectively Such findings certainly are not expected in smalls

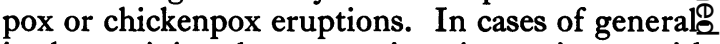
ized vaccinia also occurring in patients with pemphigus foliaceus, Fonzari (1952) noticed the same tendency to asymmetrical distribution of pocks, remarking that vaccinial lesions were fre $\overrightarrow{\vec{L}}$ quently restricted to the vaccinated arm and the neighbouring shoulder and hemithorax.

Since smallpox and a vaccination 'take' ma simultaneously develop, the time relationships the onset and disappearance of primary and secondary vaccinial lesions may have little, if any. value. However, in cases where the 'take' follows a prolonged, abnormal course the occurrence of successive crops of pocks during a long period may be an indication of vaccinia, especially if th $\overrightarrow{\mathrm{e}}$ exanthem is rather sparse. In such cases a vaco cinial ætiology is still more probable if the primary and the secondary pocks show a tendency to. necrosis. However, gangrenous smallpox lesiônక may also occur (Schamberg and Kolmer, 1928

\section{Summary}

Four cases of vaccinial eruption identified b항 virus isolation and antibody titration are preڤ sented. One case of eczema vaccinatum or Kaposi' varicelliform eruption acquired her infectiof from wardmates. The rash closely mimicke $\$$ smallpox, mainly because of the widespread distri= bution of a chronic atopic dermatitis. Anothe case exhibited gangrenous skin lesions after his vaccination and died. Clinical and histopatho logical findings in this case were typical of pros gressive vaccinia and no allergic antecedent of the patient or his family could be discovered. A thir $\$$ case had vacciniola because of the occurrence of two secondary pocks in the vicinity of the " take as well as 'inoculation' vaccinia since he showed a pock in the oral mucosa. Vaccinia virus was. recovered from the buccal pock and from salivan The fourth case suffered from asthma and infantile eczema when he acquired a vaccinial infection from an unknown source. Vaccinial pocks werê strictly confined to the eczematous patches. $A \bar{T}$ abnormal course and characteristics of the 'takeo' were noticed in these and other cases showing complications of vaccination against smallpox. The differential diagnosis of generalized vacciniæ is discussed in the light of observations made io more than 50 cases. 


\section{REFERENCES}

Angulo, J. J., and de Salles-Gomes, L. F.: unpublished observations.

Barton, R. L., and Brunsting, L. A. (r944): Kaposi's Varicelliform Eruption; Review of the Literature and Report of Two Cases of its Occurrence in Adults, Arch. Derm. Syph. (Chicago), 50, 99.

Curth, H. O., Curth, W., and Garb, J. (1948): Cutaneous Complications of Mass Vaccination in New York City, I947 (Part II), F. invest. Derm., Ir, 167.

Davidson, L. S. P., and Davies, L. J. (1943): Generalized Vaccinial Reactions in Allergic Subjects, Lancet, ii, ro3.

De Salles-Gomes, L. F., Bassoi, O. N., and Patricio, L. D. (I955): Eczema Vaccinatum with Vaccinial Lesions Strictly Confined to the Eczematous Patches, Ibid., ii, r275.

Downie, A. W., and Dumbell, K. R. (1947): Isolation and Cultivation of Variola Virus on Chorioallantois of Chick Embryos, f. Path. Bact., 69, i89.

Fonzari, M. (1952): Considerações sôbre a evolução de um caso de pênfigo foliáceo, Arch. Derm. Sif. S. Paulo, r4, 24.

Fries, J. H., BorNe, S., and BARNES, H. L. (1948): Varicelliform Eruption of Kaposi due to Vaccinia Virus Complicating Atopic Eczema, F. Pediat., 32, 532.

Gins, H. A., Hackenthal, H., and Kamentzewa, N. (1929): Experimentelle Untersuchungen über die Generalisierung des Vaccinesvirus beim Menschen und Versuchtier, Z. Hyg. Infektkr., r ro, 429.

Hall, G. F. M., Cunliffe, A. C., and Dudgeon, J. A. (1953): Prolonged Generalized Vaccinia, F. Path. Bact., 66, 25.

Hershey, F. B., and Smith, W. E. (1945): Generalized Vaccinia in an Eczematous Child; Demonstration of Virus and Comment on Kaposi's Varicelliform Eruption, Amer. F. Dis. Child., 69, 33.

Kempe, C. H. (1956): In 'Diagnostic Procedures for Virus and Rickettsial Diseases', 2nd ed. New York: American Public Health Association.

, Berge, T. O., and England, B. (1956): Hyperimmune Gammaglobulin; Source, Evaluation and Use in Prophylaxis and Treatment, Pediatrics, r8, 177.

McCarthy, K., and Downie, A. W. (1953): Serum Antibody Response in Alastrim, Lancet, i, 257.

Schamberg, J. F., and Kolmer, J. A. (r928): 'Acute Infectious Diseases', 2nd ed. Philadelphia: Lea and Febiger, p. 233 .

Sexton, G. B., Labzoffsky, N. A., and Ross, W. G. (1957): Kaposi's Varicelliform Eruption, A.M.A. Arch. Derm., 75, 361 .

Sommerville, J., NAPier, W., and Dick, A. (r95 I): Kaposi's Varicelliform Eruption; Record of an Outbreak, Brit. F. Derm., 63, 203.

WENNER, H. A. (1944): Complications of Infantile Eczema caused by the Virus of Herpes Simplex: (a) Description of the Clinical Characteristics of Unusual Eruption; and (b) Identification of an Associated Filtrable Virus, Amer. f. Dis. Child., 67, 247. 